\title{
La medición del voto ideológico en la Argentina
}

\author{
Guido Cataife ${ }^{1}$ (Argentina / Estados Unidos)
}

\begin{abstract}
Scholars have suggested that, contrary to what occurs in USA and Europe, the voters' preferences on political issues are not a major driver of the vote choice in Latin America. This article presents evidence contrary to this belief. We use two Argentine pre-electoral surveys. The first, collected in 1995, is nationally representative. The second, collected in 2009, is representative of the province of Buenos Aires. Both surveys are analyzed using a logit model with spatial ideological component. The model measures the effect of the ideological distance between voters and candidates on vote choice. In both surveys, the ideological component is found to be highly statistically significant. Further, we analyze the political relevance of the ideological vote. For the 2009 survey, political relevance is illustrated through simulation exercises. A change in the message of the three main parties yields a substantial change in their vote share, and may even alter the winner of the election. For the 1995 election, political relevance is reflected in the ability of the model to explain the party realignment observed in Argentina between the 1989 and 1995 presidential elections. Finally, we discuss the potential of the
\end{abstract}

Guido Cataife es PhD por Washington University. Actualmente se desempeña como Senior Economist en ICF International. Se especializa en el análisis estadístico y econométrico de encuestas. Entre las investigaciones del Dr. Cataife se destacan sus estudios de elecciones, comportamiento del votante y campañas electorales basados en modelos estadísticos y teoría de juegos. En el pasado, se ha desempeñado como fellow del Center for New Institutional Social Sciences (CNISS), colaborador del Center for Political Economy (Washington University), y profesor del Departamento de Economía, Escuela de Negocios, University of Louisville, en los Estados Unidos. E-mail: gcataife@icfi.com. 
logit model with spatial ideological component as a tool for the Latin American analyst and political consultant.

Síntesis: Investigadores académicos han sugerido que, a diferencia de lo que ocurre en Estados Unidos y Europa, las preferencias de los votantes en temas de políticas públicas no gozan de mayor relevancia en la decisión del voto en América Latina. El presente artículo provee evidencia en contra de dicha creencia. Se utilizan dos encuestas preelectorales argentinas. La primera, realizada en 1995, es nacionalmente representativa. La segunda, realizada en 2009, es representativa de la Provincia de Buenos Aires. Ambas encuestas son analizadas utilizando un modelo logístico con componente ideológico (también llamado "modelo espacial del voto"). Éste permite medir el efecto que la congruencia ideológica entre las preferencias de los votantes y las plataformas políticas de los diferentes partidos ejerce en la intención de voto de los encuestados. El modelo arroja alta significancia estadística del voto ideológico en ambas encuestas. La relevancia política del voto ideológico también es analizada. En la encuesta de 2009, la relevancia política se muestra con ejercicios de simulación. Una variación en el mensaje político transmitido por los tres principales partidos altera sustancialmente su porcentaje de votos, e incluso puede modificar el orden en que terminan los partidos. En la encuesta de 1995, la relevancia política del voto ideológico se refleja en la capacidad que tiene el modelo de explicar el fenómeno de realineamiento partidario observado en Argentina entre las elecciones presidenciales de 1989 y 1995. Finalmente, se discute el potencial del modelo logístico con componente ideológico como herramienta de trabajo para el analista y consultor político latinoamericano.

\section{Introducción}

El comportamiento del votante argentino es un fenómeno que ha sido poco estudiado. La importancia del voto económico (es decir, del voto que recompensa o castiga en función del crecimiento económico) ha recibido cierta atención. Sin embargo, el rol de las preferencias en materia 
de políticas públicas (de aquí en más: las preferencias políticas) ha recibido magra atención (Cataife, 2011). Podemos mencionar dos factores que han contribuido a la falta de investigación en el área. Primero, el estudio del voto basado en preferencias políticas requiere análisis estadísticos de cierta complejidad, tales como modelos econométricos logit o probit "condicionales" (Álvarez, Nagler y Bowler, 2000; Glasgow, 2001). Segundo, estudiosos académicos han manifestado su impresión de que las preferencias no juegan un rol importante en el voto latinoamericano (Stokes, 2001), trasladando el énfasis hacia el estudio de otros factores explicativos del voto, como el "voto económico" (Remmer y Gelineau, 2003) y el carisma de los candidatos (O'Donnell, 1994). Este artículo presenta evidencia significativa de que, contrariamente a la impresión prevalente entre académicos, las preferencias políticas juegan un rol significativo, tanto en términos estadísticos como en términos políticos, en la decisión del voto. El artículo ofrece dos análisis basados en dos encuestas preelectorales independientes. Una representativa de los votantes de la Provincia de Buenos Aires antes de la elección legislativa de 2009, y otra representativa del total de votantes argentinos antes de la elección presidencial de 1995. Estas dos elecciones han sido escogidas para mostrar que la relevancia del voto ideológico se manifiesta en elecciones de diversa índole -una elección es legislativa, la otra presidencial- y en períodos históricos distintos, separados por 14 años.

Ambas encuestas son analizadas utilizando el modelo logístico conocido como modelo espacial del voto (Dow y Endersby, 2004). Tal nombre se deriva del hecho de que el modelo logístico incorpora la distancia "espacial" entre el votante y los candidatos en varias dimensiones ideológicas, tales como el rol del Estado en la economía, la política contra la inseguridad, los derechos de las minorías, etc. 
El modelo permite medir el efecto que la incongruencia ideológica (o "distancia espacial") entre las preferencias de los votantes y las plataformas políticas de los diferentes partidos ejerce en la intención de voto de los encuestados.

Se presentan tres argumentos en contra de la hipótesis de que el voto ideológico no tiene relevancia. Primero, se muestra que los coeficientes del modelo asociados a la distancia ideológica entre votantes y candidatos tienen relevancia estadística a niveles convencionales de significancia. Segundo, se demuestra que, además de ser estadísticamente relevante, el voto ideológico es políticamente relevante, al registrarse sustanciales variaciones en el porcentaje de votos de cada candidato (en base a la encuesta de 2009) en reacción a cambios simulados en la posición de los candidatos imputados a los votantes. Tercero, se provee adicional evidencia de la relevancia política del voto ideológico al mostrarse que los coeficientes del modelo logístico estimados en base a la encuesta de 1995 facilitan la interpretación del período 1989-1995, el cual trajo grandes cambios en el sistema político argentino. El modelo permite visualizar tales cambios como el resultado de un "realineamiento partidario" producido por el Plan de Convertibilidad, lo cual se encuentra en línea con argumentos teóricos previos (Schofield y Cataife, 2007; Gervasoni, 1998).

Finalmente, el artículo sugiere que, dada la evidencia de que el voto ideológico es relevante en la decisión del voto, el modelo logístico con componente espacial constituye una refinada y eficaz herramienta para el analista político y consultor electoral latinoamericano. El modelo ofrece al consultor en temas de opinión pública una mayor precisión en la respuesta a los interrogantes típicos, y la posibilidad de elaborar nuevas estrategias de campaña electoral, como el microtargeting de votantes. 


\section{Elecciones parlamentarias 2009}

El primer análisis utiliza datos de una encuesta recolectada por la consultora Ibarómetro, seis semanas antes de las elecciones parlamentarias argentinas de junio de 2009. A diferencia de otras elecciones parlamentarias, las elecciones de junio de 2009 gozaron de gran trascendencia en los medios y entre el público en general, por dos razones. Primero, la presidenta Cristina Fernández de Kirchner venía sufriendo un marcado deterioro en las encuestas de opinión pública. Segundo, existían fuertes debates públicos en torno a la confrontación del gobierno con varios sectores de la sociedad, incluyendo el agro y el mayor conglomerado mediático del país.

La encuesta comprende únicamente votantes de la Provincia de Buenos Aires. Dado que la encuesta fue diseñada con el fin específico de poder estimar un modelo logístico avanzado, cuenta con las propiedades ideales para investigar el rol de las preferencias en el voto de los argentinos. En particular, permite estimar el peso que la distancia ideológica entre votantes y candidatos (en materia de políticas públicas) tiene en la decisión del voto, controlando estadísticamente por variables que influyen el voto. ${ }^{2}$ Para cada tema de política pública (seguridad, rol del Estado, etc.), la distancia ideológica del votante respecto a un candidato se obtiene como (el valor absoluto de) la diferencia entre la posición que el votante declara como su preferida en una escala del 1 al 7 y la posición promedio en que los votantes ubican al candidato. Así, por ejemplo, un votante que en la escala 1-7 (donde 1 significa preferencia firme por una política de mano dura contra el

En el estudio, se controla por: educación, edad, sexo, área geográfica, identificación partidaria y variables proxy del nivel socioeconómico (posesión de teléfono, uso de hospital público, obra social, o medicina privada). 
crimen y 7 significa preferencia firme por una política de prevención y educación contra el crimen) dice ubicarse en el 2, se encontraría a sólo 1.73 unidades de distancia con respecto a Francisco De Narváez, a quien los encuestados en promedio ubican en 3.73; y a 2.61 unidades de distancia con respecto a Margarita Stolbizer, a quien los encuestados en promedio ubican en 4.61 .

La ilustración 1 muestra la distribución de las preferencias de los votantes de la Provincia de Buenos Aires en un espacio bidimensional de políticas públicas, conformado por la política de seguridad (eje vertical) y la política de creación de empleo (eje horizontal). La ilustración muestra los contornos de máxima densidad de votantes y la posición percibida en promedio para las tres listas principales de las elecciones legislativas 2009. Se puede observar que el grueso de los votantes tiene lo que podríamos denominar "preferencias extremas". En efecto, son muy pocos los encuestados que adoptan posiciones intermedias, como 2, 3, 4, 5, ó 6 . La gran mayoría prefiere un Estado que combate el crimen con "prevención y educación" únicamente (en vez de una política que combine prevención con firmeza en la aplicación de la ley) y un Estado máximamente activo en la generación de empleo (en vez de una política que combine intervencionismo con la autorregulación del mercado de trabajo). Aunque el extremismo de estas preferencias es llamativo, no hay razones para dudar de su veracidad, por dos motivos. Primero, preferencias con un similar nivel de extremismo se desprenden de la encuesta nacionalmente representativa recolectada en Argentina en 2010 por Latin American Public Opinion Project, coordinado por Vanderbilt University. Segundo, dado que el grueso de los encuestados ubica a los candidatos en posiciones intermedias (2, 3, 4 y 6 ), no hay razones para pensar que las preguntas relativas a la ubicación en la escala 1-7 hayan sido malinterpretadas por los encuestados. 


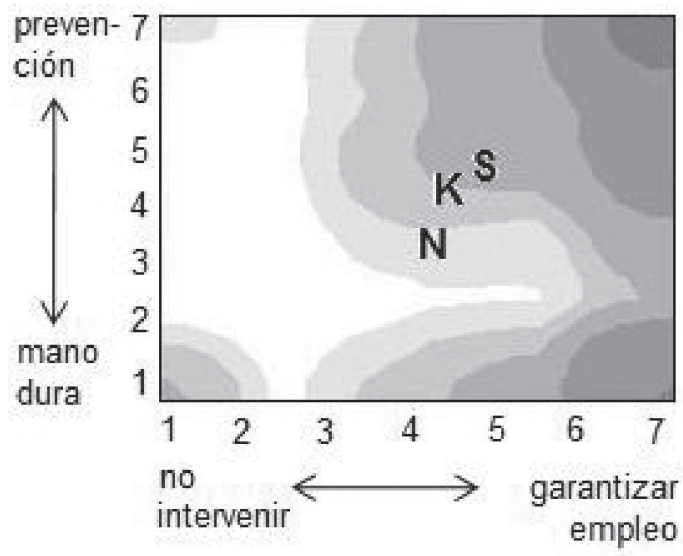

Ilustración 1. Distribución de votantes en un espacio bidimensional de política pública graficada como contornos de máxima densidad de votantes al 1, 5, 10, 25 y 50\%. Los puntos K, N y S representan la media de la percepción de los encuestados para las listas encabezadas por Néstor Kirchner, Francisco de Narváez y Margarita Stolbizer, respectivamente.

El modelo logístico busca el conjunto de parámetros que con mayor probabilidad explica la distribución de microdatos recolectada por la encuesta. Es decir, utiliza estimación por máxima verosimilitud. En vez de preguntarle a los individuos cuáles son los factores que determinan su voto, les preguntamos por quién piensan votar, cuáles son sus características sociodemográficas y cuáles son sus preferencias políticas. Utilizamos K, Ny S, para denotar las fórmulas encabezadas por Kirchner, Narváez y Stolbizer, respectivamente. El modelo logístico con tres candidatos $(\mathrm{K}, \mathrm{N}, \mathrm{S})$ se centra en la probabilidad de voto del votante $i$ condicional en el vector de variables independientes $x$ $=\{$ variables sociodemográficas, percepción de atributos de los candidatos, distancia ideológica entre votantes y 
candidatos\}, la que toma la siguiente forma para cada candidato:

$$
\begin{aligned}
& \operatorname{Pr}\left(\text { voto }_{i}=N\right)=\frac{\exp \left(\beta_{0}+x_{i} \beta_{N}\right)}{1+\exp \left(\beta_{0}+x_{i} \beta_{N}\right)+\exp \left(\beta_{0}+x_{i} \beta_{S}\right)}, \\
& \operatorname{Pr}\left(\text { voto }_{i}=S\right)=\frac{\exp \left(\beta_{0}+x_{i} \beta_{S}\right)}{1+\exp \left(\beta_{0}+x_{i} \beta_{N}\right)+\exp \left(\beta_{0}+x_{i} \beta_{S}\right)}, \\
& \operatorname{Pr}\left(\text { voto }_{i}=K\right)=\frac{1}{1+\exp \left(\beta_{0}+x_{i} \beta_{N}\right)+\exp \left(\beta_{0}+x_{i} \beta_{S}\right)},
\end{aligned}
$$

Donde K es el candidato de referencia, cuya utilidad debe ser normalizada a cero para poder identificar los parámetros del modelo.

El procedimiento de máxima verosimilitud estima el peso relativo (denotado con la letra griega beta) que cada variable independiente incluida en el vector $x$ ejerce en la decisión del voto. En otras palabras, el modelo analiza cómo los argentinos votan sin preguntarles por qué votan como votan, lo cual reduce sesgos en las respuestas y evita supuestos poco confiables en términos de la capacidad de introspección de los encuestados. El modelo fue estimado utilizando el software NLOGIT.

Los coeficientes estimados por el modelo logístico (en base a la encuesta de Ibarómetro en la Provincia de Buenos Aires) se encuentran disponibles en Cataife (2011). Son omitidos aquí por carecer de una interpretación directa, a diferencia de la regresión lineal por mínimos cuadrados ordinarios, cuyos coeficientes pueden ser interpretados como efectos marginales. La distancia ideológica en el tema de la seguridad surge como un factor de elevada significancia estadística (al 1\% en una muestra de 1.229 
individuos) en la decisión del voto. En el presente artículo utilizamos dichos coeficientes y los mismos microdatos utilizados en su estimación (microdatos correspondientes a la encuesta de la Provincia de Buenos Aires) para simular cambios en la estrategia política de los candidatos que encabezaron las tres principales listas (Narváez-Solá, Stolbizer-Alfonsín, Kirchner-Scioli) en la elección legislativa de 2009 correspondiente a la Provincia de Buenos Aires. El objetivo es investigar cuánto varía la intención de voto de cada candidato cuando cambian las plataformas políticas. Específicamente, simulamos cambios en la política de seguridad de los candidatos, entendida como el grado de "mano dura" versus "prevención y educación" en la escala 1-7. La descripción técnica del procedimiento utilizado para simular cambios en la posición de los candidatos en el espacio de políticas públicas, y medir su impacto en el porcentaje de votos de cada partido utilizando los coeficientes de un modelo logístico, se encuentra disponible en Álvarez y Nagler (1995) y Schofield y Sened (2006).

Tabla 1. Simulación de porcentaje de votos bajo distintos escenarios

\begin{tabular}{|c|c|c|c|c|}
\hline & Muestra (\%) & Escenario I (\%) & Escenario II (\%) & Escenario III (\%) \\
\hline Narváez-Solá & 38,3 & 44,2 & 36,2 & 35,6 \\
\hline Stolbizer-Alfonsín & 23,9 & 21,6 & 24,0 & 29,4 \\
\hline Kirchner-Scioli & 37,9 & 34,3 & 40,8 & 35,6 \\
\hline
\end{tabular}

La tabla 1 presenta cuatro columnas con porcentajes de voto estimados para cada uno de los tres partidos principales en la elección legislativa de 2009 por la Provincia de Buenos Aires. La columna encabezada "Muestra" corresponde a los porcentajes de voto reales que se desprenden de la muestra. Las restantes columnas corresponden a ejercicios de simulación. Éstos fueron realizados en base a 
la estimación de coeficientes del modelo logístico reportado en Cataife (2011). ${ }^{3}$ De la columna "Muestra" se desprende que, utilizando la posición ideológica de los candidatos reportada por los encuestados, Narváez-Solá es la lista ganadora (38,3\% de los votos). Luego siguen Kirchner-Scioli (37,9\% de los votos) y Stolbizer-Alfonsín (23,9\%). ${ }^{4}$ Los escenarios I, II y III simulan el efecto de cambios en la posición de los candidatos en el tema "política contra el crimen". La posición ideológica de los candidatos percibida por los encuestados en el tema seguridad (donde 1 representa

La variable dependiente de cada ecuación de dicho modelo es la probabilidad de voto por cada partido. Las variables independientes miden: sexo, educación, área geográfica, uso de hospital público, obra social o medicina prepaga (proxy de ingreso), posesión de teléfono (proxy de ingreso), identificación partidaria, evolución de la situación económica del encuestado en el último año, percepción de fórmula Kirchner-Scioli en materia de: I. Liderazgo; II. Conocimiento de los problemas de la Provincia de Buenos Aires; III. Interés por resolver los problemas de la gente. Las restantes variables dependientes miden la distancia ideológica de los encuestados respecto a cada candidato en cada una de las siguientes políticas de Estado: seguridad, servicios sociales, Estado como creador de empleo. Para una descripción en mayor detalle de las variables véase Cataife (2011).

$4 \quad$ Para cada partido, el porcentaje de voto estimado por el modelo (38,3\%; 37,9\%; y 23;9\% para Narváez-Solá, Kirchner-Scioli y Stolbizer-Alfonsín, respectivamente) es mayor al porcentaje obtenido en las elecciones (34;5\%; $32 \% ;$ y $21,5 \%$, respectivamente). En parte, esta discrepancia se debe a que el modelo usa la probabilidad estimada de voto por los candidatos (en vez de la intención de voto reportada por el encuestado) para calcular los porcentajes de voto obtenidos por los partidos, lo cual da lugar a cierto error predictivo. Pero, en su gran mayoría, esta discrepancia se debe a que en la estimación del modelo hemos excluido deliberadamente a los partidos menores con el objeto de mejorar la bondad de ajuste de la estimación. Lógicamente, al excluir varios partidos, los porcentajes de los tres partidos considerados terminan siendo mayores a los reales. De hecho, el modelo predice notablemente bien los porcentajes de voto relativos de los candidatos. Para ver esto, nótese que el porcentaje de voto estimado de Narváez-Solá relativo al estimado de Kirchner-Scioli es 1,01, y relativo al estimado de Stolbizer-Alfonsín es 1,60 , cuando los porcentajes relativos obtenidos en la elección son 1,07 y 1,60 , respectivamente. 
firme mano dura y 7 representa únicamente prevención y educación) es: Narváez-Solá = 3,73; Kirchner-Scioli = 4,43; Stolbizer-Alfonsín $=4,61 .{ }^{5}$ El escenario I simula el siguiente reposicionamiento de los candidatos: Narváez-Solá $=5$; Kirchner-Scioli = 1; Stolbizer-Alfonsín $=2$. El escenario II simula el siguiente cambio en la posición de los candidatos: Narváez-Solá = 1; Kirchner-Scioli = 5; Stolbizer-Alfonsín = 2. Dada la distribución de preferencias de los encuestados en materia de seguridad, la posición 5 es claramente ventajosa, ya que coincide con el votante mediano en tal dimensión (por la misma razón, la posición 1 es claramente desventajosa). Nótese que los escenarios I y II rotan la posición de las dos primeras fórmulas (Narváez-Solá y Kirchner-Scioli) en la dimensión "seguridad", manteniendo la fórmula Stolbizer-Alfonsín fija. Este sencillo ejercicio muestra la magnitud del efecto de la ideología (entendida como la discrepancia entre votantes y candidatos en cuanto a preferencias por políticas públicas) en el voto: la fórmula que se posiciona en 5 obtiene el primer lugar en la elección, y la fórmula que se posiciona en 1 obtiene el segundo lugar. La rotación de 1 a 5 implica una diferencia de 8 puntos porcentuales para Narváez-Solá y de 6,5 puntos porcentuales para Kirchner-Scioli. Diferencias de tal magnitud pueden definir incluso elecciones no tan reñidas y, claro está, pueden generar diferencias harto significativas en términos de bancas parlamentarias ganadas por un partido u otro en elecciones parlamentarias. El escenario III simula el siguiente cambio en la posición de los candidatos: Narváez-Solá $=1$; Kirchner-Scioli $=1$; Stolbizer-Alfonsín = 5, lo cual ubica a Stolbizer-Alfonsín en una posición ventajosa con respecto a sus contrincantes. Aunque ello mejora significativamente la performance

Siguiendo la literatura, la posición ideológica de los candidatos percibida por los encuestados es la media muestral. 
electoral de Stolbizer-Alfonsín con respecto a los valores "reales" reflejados en la columna "Muestra", no es suficiente para alcanzar el segundo puesto.

\section{El realineamiento partidario en Argentina 1989-1995}

La fórmula presidencial del PJ (Menem-Duhalde) ganó la elección presidencial de 1989 en Argentina con una plataforma fuertemente redistribucionista, la cual incluía el "salariazo" y la "revolución productiva". La clase trabajadora, identificada con el PJ, apoyó a Menem y le dio la victoria. La plataforma del principal partido opositor, la UCR (AngelozCasella), consistía en el llamado "lápiz rojo", el que aludía a la intención de reducir el aparato estatal en un intento de austeridad fiscal para frenar la inflación y generar estabilidad macroeconómica. Menem ganó la elección con el $47,5 \%$ de los votos, y Angeloz obtuvo el segundo lugar con $37 \%$. Poco después de ser nombrado presidente, Menem implementó un paquete de políticas, incluido el Plan de Convertibilidad, incongruente con su plataforma electoral. Cinco años después de la implementación del Plan, Menem tuvo la oportunidad de presentarse a la reelección. Esta vez, su plataforma era apoyada por una nueva coalición electoral que incluía a parte de la clase media y clase alta. Esta nueva coalición posibilitó la victoria de Menem al contrarrestar la pérdida de votos de los sectores populares. A continuación, mostramos cómo el modelo logístico con componente ideológico ofrece claves para la interpretación del período 1989-1995, el cual trajo grandes cambios en el sistema político argentino. El modelo permite visualizar dichos cambios como el resultado de un "realineamiento partidario" producido por el Plan de Convertibilidad. 
Para ello implementamos un modelo logístico del voto con componente ideológico utilizando una encuesta preelectoral recolectada en marzo de 1995 por Mansilla, Delich y Asociados, depositada en Roper Center. La encuesta, nacionalmente representativa, fue suministrada a 1.600 individuos. Contiene información sobre: el voto del encuestado en las elecciones presidenciales de 1989; la intención de voto del encuestado para las elecciones presidenciales de mayo 1995; características sociodemográficas del encuestado; y su punto de vista sobre cuáles (dado un conjunto de opciones) son los objetivos de política prioritarios a cumplir por los candidatos presidenciales. ${ }^{6} \mathrm{~A}$ diferencia de la encuesta de la Provincia de Buenos Aires utilizada en la sección anterior, esta encuesta no incluye preguntas que permitan medir la posición del votante (en una escala tipo Likert) en los mencionados temas prioritarios. Por esta razón, no es posible estimar el modelo espacial canónico (Dow y Endersby, 2004) tal y como fue desarrollado, sin modificaciones. De todos modos, la dimensionalidad de la política argentina y la posición de los distintos partidos pueden inferirse de la estimación de un modelo logístico que, en vez de incorporar la distancia ideológica entre el votante y el candidato, incorpore la importancia que cada votante le asigna a cada "tema" de política. Tal modelo nos permite calcular el "beneficio" que cada partido obtiene por enfatizar uno u otro tema de política en sus plataformas. Dicho beneficio puede ser medido en términos del cambio en la probabilidad de voto (de cada votante, cada grupo de votantes, o en promedio) cuando se simulan cambios en las prioridades de política adoptadas por los candidatos.

La pregunta textual es: "Le voy a presentar un listado de objetivos a cumplir por los candidatos presidenciales. Por favor, dígame, ¿cuáles considera más importantes? (sólo 2)." 
Tal modelo logístico constituye una buena aproximación al modelo logístico espacial canónico.

Los tres partidos principales en las elecciones de 1995 fueron el PJ (fórmula presidencial encabezada por Carlos Menem), la UCR (Horacio Massaccesi) y el recientemente formado partido de centro-izquierda FREPASO (José Bordón). Las variables incluidas en el modelo se encuentran descriptas y sintetizadas en la tabla 2. Las variables dicotómicas estabilidad, crimen, justsoc y pymes toman el valor 1 si el encuestado considera que la política en cuestión está entre las dos prioridades de la plataforma política del candidato, y 0 si considera que no lo está. La variable estabilidad se refiere a una política de estabilidad macroeconómica, la variable crimen se refiere a una política contra la inseguridad, la variable justsoc se refiere a una política que genere mayor justicia social, y la variable pymes se refiere a una política que apoye a las pequeñas y medianas empresas nacionales. Los encuestados pueden reportar ninguna, una o dos prioridades de política para cada fórmula presidencial de una lista de 10 opciones (además de la opción NS / NR), que incluye las cuatro detalladas. ${ }^{7}$ La regresión logística controla las siguientes características sociodemográficas del encuestado: sexo, grupo etario, nivel educativo y ciudad de residencia. Los coeficientes asociados a estas variables no son reportados para conservar espacio. Como en todo modelo logístico, la utilidad asociada a una de las opciones debe ser normalizada a cero. En este caso, normalizamos respecto a FREPASO. Los parámetros estimados del modelo logístico para la elección presidencial de 1995 se reportan en la tabla 3.

Además de estabilidad, crimen, justsoc y pymes, la encuesta ofrece a los encuestados la posibilidad de seleccionar otras opciones, incluyendo políticas de salud, educación, crecimiento, empleo y corrupción. Estas últimas fueron excluidas del análisis tras arrojar insignificancia estadística en la regresión, a los efectos de reducir multicolinearidad. 
Tabla 2

Descripción de variables: intención de voto 1989, voto 1995 y voto por preferencias

\begin{tabular}{|c|c|c|c|}
\hline Variable & Descripción & Media & $\begin{array}{c}\text { Desvío } \\
\text { estándar }\end{array}$ \\
\hline Voto95_PJ & $\begin{array}{c}\text { Intención de voto por PJ en elecciones } \\
1995 \text { (dicotómica) }\end{array}$ & 0.479 & 0.499 \\
\hline Voto95_UCR & $\begin{array}{c}\text { Intención de voto por UCR en eleccio- } \\
\text { nes 1995 (dicotómica) }\end{array}$ & 0.160 & 0.366 \\
\hline $\begin{array}{c}\text { Voto95__ } \\
\text { FREPASO }\end{array}$ & $\begin{array}{c}\text { Intención de voto por FREPASO en } \\
\text { elecciones 1995 (dicotómica) }\end{array}$ & 0.360 & 0.480 \\
\hline Voto89_PJ & $\begin{array}{c}\text { Votó por PJ en 1989 (dicotómica) } \\
\text { Voto89_UCR }\end{array}$ & 0.405 & 0.491 \\
\hline Voto89_UCEDÉ por UCR en 1989 (dicotómica) & 0.244 & 0.429 \\
\hline Voto89_otro & $\begin{array}{c}\text { Votó por por UCEDE en 1989 (dicotómica) } \\
\text { 1989 (dicotómica) }\end{array}$ & 0.031 & 0.174 \\
\hline Estabilidad & $\begin{array}{c}\text { Preservar estabilidad económica es } \\
\text { uno de los dos objetivos principales } \\
\text { (dicotómica) }\end{array}$ & 0.108 & 0.311 \\
\hline Crimen & $\begin{array}{c}\text { Reducir el crimen es uno de los dos } \\
\text { objetivos principales (dicotómica) }\end{array}$ & 0.064 & 0.246 \\
\hline Justsoc & $\begin{array}{c}\text { Incrementar justicia social es uno } \\
\text { de los dos objetivos principales } \\
\text { (dicotómica) }\end{array}$ & 0.087 & 0.282 \\
\hline Pymes & $\begin{array}{c}\text { Apoyar a las pequeñas y medianas } \\
\text { empresas es uno de los dos objetivos } \\
\text { principales (dicotómica) }\end{array}$ & 0.064 & 0.246 \\
\hline
\end{tabular}

De la tabla 2 se desprende que, hacia 1995, la primera prioridad reportada por los votantes era la estabilidad económica; un $10,8 \%$ de los encuestados la considera prioritaria. Le siguen justicia social $(8,7 \%)$, y recién luego crimen y pymes $(6,4 \%)$. Para entender el efecto que estas 
prioridades ejercieron sobre el voto estimamos el modelo logístico, cuyos resultados son reportados en la tabla 3.

Tabla 3. Modelo logístico

Variable dependiente: intención de voto en la elección presidencial de 1995

\begin{tabular}{|c|c|c|c|c|}
\hline & \multicolumn{2}{|c|}{ PJ (Menem) } & \multicolumn{2}{|c|}{ UCR (Massaccesi) } \\
\hline Variable & Coeficiente & SE & Coeficiente & SE \\
\hline Voto89_UCR & $-1.960 * * *$ & 0.199 & $1.870^{* * *}$ & 0.275 \\
\hline Voto89_otro & $-0.827^{* * *}$ & 0.156 & $0.962^{* * *}$ & 0.284 \\
\hline Estabilidad & $0.905^{* * *}$ & 0.905 & 0.397 & 0.322 \\
\hline Crimen & $0.624^{*}$ & 0.290 & 0.379 & 0.399 \\
\hline Justsoc & $-0.457^{*}$ & 0.225 & $-0.998^{* *}$ & 0.364 \\
\hline Pymes & -0.246 & 0.266 & -0.111 & 0.352 \\
\hline Constant & $1.451^{* * *}$ & 0.371 & $-1.321^{*}$ & 0.531 \\
\hline Control:edad & Sí & Sí & Sí & Sí \\
\hline Control: sexo & Sí & Sí & Sí & Sí \\
\hline $\begin{array}{l}\text { Control: } \\
\text { educación }\end{array}$ & Sí & Sí & Sí & Sí \\
\hline $\begin{array}{l}\text { Efectos fijos: } \\
\text { Ciudad }\end{array}$ & Sí & Sí & Sí & Sí \\
\hline $\begin{array}{r}\text { otas: La utilic } \\
1\end{array}$ & $\begin{array}{l}\text { REPASC } \\
\mathrm{hi} 2=43\end{array}$ & 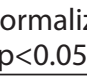 & $\begin{array}{l}\mathrm{V}=13 \\
{ }^{*} \mathrm{p}<\end{array}$ & $=0.16$ \\
\hline
\end{tabular}

Como se observa en la tabla 3 , los coeficientes +1.87 y -1.96, asociados a voto89_UCR para la intención de voto a la UCR y al PJ, respectivamente, no arrojan sorpresas. Haber votado para la UCR en la elección presidencial de 1989 incrementa la utilidad de votar por dicho partido en 1995 y decrece la utilidad de votar por el PJ. La variable voto89_otro incluye todos los encuestados que no votaron por el PJ o la UCR en el 1989. Estos encuestados votaron por otros partidos o no votaron. La variable de referencia en la regresión es voto89_PJ. Estas tres variables también funcionan como proxies de identificación partidaria. La 
constante, 1.451, es estadísticamente significativa y refleja la ventaja promedio del PJ sobre la UCR y el FREPASO a la hora de decidir el voto, en términos de todas la variables no incluidas en la regresión por falta de datos, tales como carisma, liderazgo, peso del aparato partidario, etc.

Nuestro mayor interés se centra en los coeficientes asociados a las preguntas de política, dado que nos permiten comprender, para cada perfil de votante, el peso relativo de los distintos factores que influyen el voto. Más aun, como veremos, en este caso el modelo presenta fuerte evidencia a favor de análisis teóricos previos que sugieren que la Ley de Convertibilidad, implementada en el primer mandato de Menem, al combatir eficazmente la inflación indujo un realineamiento partidario en la política nacional argentina, producido entre 1989 y 1995 (Schofield y Cataife, 2007; Gervasoni, 1998). ${ }^{8}$ Menem ganó la elección de 1989 con una plataforma fuertemente redistributiva ("salariazo" y "revolución productiva"), congruente con los valores de "justicia social" tradicionalmente asociados al PJ. Nuestro modelo logístico muestra que, seis años más tarde, Menem ganó la reelección en base a una plataforma percibida por los votantes de manera radicalmente opuesta. Como muestran los coeficientes estimados en la tabla 3, en 1995 los votantes ya no consideraban al PJ como el partido de la justicia social. De hecho, el modelo sugiere que el considerar que "justicia social" es uno de los dos objetivos prioritarios reduce la utilidad que el votante obtiene de votar

\footnotetext{
Dicho argumento se fundamenta en la idea de que existen al menos dos "dimensiones" en la política argentina. La primera podría llamarse "política externa" e incluye un conjunto de variables que afectan el tipo de cambio real efectivo, especialmente: tipo de cambio nominal, inflación, impuestos a la exportación. Estas tres variables están íntimamente relacionadas y tienen un fuerte impacto sobre el consumo interno de bienes transables. Y la segunda podría llamarse "redistribución", e incluye transferencias entre clases sociales y provisión de bienes públicos (en un sentido amplio del término) como educación, salud, vivienda, etc.
} 
por PJ con respecto al partido de referencia (FREPASO). Para ver esto, nótese que el coeficiente estimado asociado a la variable justsoc para el PJ, -0.457, es negativo y estadísticamente significativo al 5\%. Por ejemplo, el uso de los coeficientes para estimar la probabilidad de voto sugiere que un votante con características sociodemográficas medianas que ha votado por un partido que no es el PJ ni la UCR en las elecciones presidenciales de 1989 tiene una probabilidad 0.28 de votar por el FREPASO en 1995, si no considera que la justicia social es un tema prioritario. Pero esta probabilidad salta a 0.41 cuando el mismo votante sí considera que la justicia social es un tema prioritario. Ello equivale a un incremento de $46 \%$ en la probabilidad de apoyar al FREPASO, partido opositor del PJ. ${ }^{9}$

Por otro lado, la estimación muestra que en 1995 el PJ estaba identificado de forma clara con la estabilidad macroeconómica. Más precisamente, el modelo sugiere que considerar la estabilidad macroeconómica como una política prioritaria incrementa la utilidad que el votante deriva de votar al PJ respecto al FREPASO. Para ver esto, nótese que el coeficiente estimado asociado a la variable estabilidad para el PJ, +0.905 , es positivo y estadísticamente significativo. La UCR, cuya propuesta central en la campaña presidencial de 1989 era el "lápiz rojo" para reducir el aparato estatal y ganar austeridad fiscal, es ahora vista como una alternativa inferior al PJ en este respecto. Para ver esto, obsérvese que el coeficiente asociado a la UCR, +0.379 , no es estadísticamente significativo, lo que sugiere que los votantes consideran en promedio a la UCR y al FREPASO como "equivalentes" en su capacidad para garantizar estabilidad macroeconómica, e inferiores al PJ en este respecto. Los cómputos basados en estas estimaciones

El procedimiento técnico para calcular la probabilidad de voto utilizando los coeficientes del modelo logístico se detalla en McFadden (1973). 
sugieren que un votante con características sociodemográficas medianas que haya votado por un partido que no es el PJ ni la UCR en las elecciones presidenciales de 1989 tiene una probabilidad de votar por el PJ en 1995 de 0.50 cuando no considera que la estabilidad macroeconómica es una política prioritaria. Pero esta probabilidad salta al 0.67 cuando el mismo votante sí considera que la estabilidad macroeconómica es un tema prioritario. Ello representa un incremento del $34 \%$ en la probabilidad de respaldar al PJ.

\section{Conclusión}

El modelo logístico con componente ideológico permite medir el peso relativo de los distintos factores que influyen en el voto de cada perfil de votante. Por tal motivo, constituye la herramienta más adecuada para testear empíricamente la impresión sostenida por académicos de que el voto ideológico, entendido como el peso de la congruencia entre las preferencias de política pública de votantes y candidatos en la decisión de voto, tiene escasa relevancia en América Latina. El presente artículo exhibe estimaciones en base a dos encuestas correspondientes a muestras y años distintos. En ambos casos, el voto ideológico alcanza relevancia estadística. Dado que la relevancia estadística es condición necesaria pero no suficiente para mostrar relevancia política, el artículo complementa el análisis de dos maneras. Primero, por medio de simulaciones se demuestra que varios posibles cambios en las plataformas políticas de los candidatos (percibidas por los votantes) arrojan sustanciales variaciones en los porcentajes de voto esperado por los partidos. Segundo, los coeficientes estimados en base a datos de 1995 ofrecen la llave para la comprensión del convulsionado período 19891995, al mostrar que en los ojos de los votantes los partidos 
políticos sufrieron una brusca realineación en un espacio político bidimensional conformado por la política social (justicia social) y la política macroeconómica (estabilidad). La medida en que dicho realineamiento haya contribuido a la proliferación de terceros partidos en un sistema hasta entonces bipartidario es un tópico de interés que queda en la agenda de investigación. No obstante, el autor se atreve a sugerir la hipótesis de que, luego del reposicionamiento del PJ tras la Ley de Convertibilidad, la UCR no supo reubicarse estratégicamente en el espacio bidimensional, facilitando la aparición de ubicuos terceros partidos. De hecho, el testeo empírico de esta hipótesis (que es indudablemente una entre muchas otras posibles) también podría realizarse a través de un modelo logístico que mida la posición de los partidos y su relevancia estadística en la decisión del voto en las elecciones posteriores a 1995. O, alternativamente, podría testearse indirectamente simulando la posición óptima de la UCR frente a la nueva posición del PJ en el espacio bidimensional. Tales estudios son factibles y han sido realizados para otros países (Schofield y Sened, 2006).

Finalmente, cabe enfatizar la relevancia del modelo logístico con componente ideológico como herramienta para el analista y consultor político. Al mostrar que el voto ideológico tiene relevancia en la Argentina (y presumiblemente también la tenga en otros países latinoamericanos), el presente artículo señala nuevos horizontes para los profesionales del estudio de la opinión pública en la región. Para lograr efectividad, el asesor político y el consultor de marketing político necesitan información sobre las preferencias ideológicas de los votantes y su percepción de las plataformas de los candidatos. El modelo logístico es la herramienta más apropiada para tales fines, por su nivel de refinamiento y su confiabilidad. Asimismo, el modelo logístico es una de las herramientas fundamentales utilizadas en el diseño de estrategias de microtargeting (Malchow, 
2008), las cuales constituyen un elemento indispensable en las campañas políticas realizadas en la actualidad en Estados Unidos y Europa. El microtargeting consiste en enviar mensajes con contenido político bien específico, customizados en base a las preferencias ideológicas de cada perfil de votante, con la finalidad de incrementar la probabilidad de que el votante apoye al candidato (el modelo logístico es la herramienta más adecuada para el microtargeting porque permite estimar la probabilidad de que un determinado votante apoye a un candidato, así como simular cambios en dicha probabilidad, tal como hemos hecho en la segunda sección del presente artículo.) Hasta ahora ignorado en América Latina (por considerarse de un modo equivocado, según argumenta este artículo, que el votante latino basa su voto sólo en el carisma de los líderes y la performance de la macroeconomía), el microtargeting puede ser el gran nuevo aliado del consultor político en la región.

\section{Bibliografía}

Álvarez, M. y Nagler, J. (1995), “Economics, Issues, and the Perot Candidacy: Voter Choice in the 1992 Presidential Election", American Journal of Political Science, núm. 39, pp. 714-744.

Álvarez, M.; Nagler, J.; y Bowler, S. (2000), "Issues, Economics, and the Dynamics of Multiparty Elections: The British 1987 General Election", American Political Science Review, núm. 94, pp. 131-150.

Cataife, G. (2011), "An Integrated Model of Vote Choice in Argentina, 2009", Latin American Politics and Society, en imprenta.

Dow, J. y Endersby, J. (2004), "Multinomial Probit and Multinomial Logit: A Comparison of Choice Models 
for Voting Research", Electoral Studies, núm. 23, pp. 107-122.

Gervasoni, C. (1998), “Del distribucionismo al neoliberalismo: los cambios en la coalición electoral peronista durante el gobierno de Menem", artículo presentado en la Conferencia 2008 de la Latin American Studies Association.

Glasgow, G. (2001), "Mixed Logit Models for Multiparty Elections", Political Analysis, núm. 9, pp. 116-136.

Malchow, Hal (2008), Political Targeting, de próxima publicación.

McFadden, D. (1973), "Conditional Logit Analysis of Qualitative Choice Behavior”, en Zarembka, P. (ed.), Frontiers in Econometrics, Nueva York, Academic Press.

O'Donnell, G. (1994), "Delegative Democracy", Journal of Democracy, núm. 5, pp. 55-69.

Remmer, K. y Gelineau, G. (2003), "Subnational Electoral Choice: Economic and Referendum Voting in Argentina, 1983-1999", Comparative Political Studies, núm. 36, pp. 801-821.

Schofield, N. y Cataife, G. (2007), "A Model of Political Competition with Activists with an Application to the Elections of 1989 and 1995 in Argentina", Mathematical Social Sciences, núm. 53, pp. 213-231.

Schofield, N. y Sened, I. (2006), Multiparty Democracy, Cambridge, Cambridge University Press.

Stokes, S. (2001), Mandates and Democracy: Neoliberalism by Surprise in Latin America, Cambridge, Cambridge University Press. 